\title{
EXPLORING MODAL VERBS CONVEYING POSSIBILITY IN ACADEMIC DISCOURSE
}

\author{
Petra Huschová
}

\begin{abstract}
This paper explores the occurrence and use of the English modal verbs CAN/COULD and MAY/MIGHT conveying possibility meanings in academic texts dealing with linguistics and attempts to reveal the contextual factors determining the interpretation of the verbs. The paper discusses the semantic components of the examined modal verbs in relation to syntactic co-occurrence patterns and stylistic variation, focusing on the factors governing the distribution and usage of their epistemic and root possibility readings. Finally, the paper comments on the possibility readings of CAN/COULD and MAY/MIGHT which can be employed as hedging devices.
\end{abstract}

\section{Key words}

CAN/COULD; MAY/MIGHT, epistemic possibility, root possibility, academic discourse, hedging

\section{Introduction}

This paper deals with the use of modal verbs expressing possibility, i.e. the verbs CAN/COULD and MAY/MIGHT, focusing primarily on the role of context in interpreting their meanings. It aims to distinguish between their root and epistemic possibility readings, to investigate the contexts in which root or epistemic readings prevail, and to discuss the uses of the analysed modal verbs which can be employed as hedging devices.

As for the excerpted material, the analysis is based on a small-scale corpus of 400 contextualized occurrences of CAN/COULD and MAY/MIGHT, which have been drawn from five English academic texts dealing with linguistics, namely from (a) Register, Genre and Style by Douglas Biber and Susan Conrad; (b) Relevance and Linguistic Meaning by Diane Blakemore; (c) Corpus Stylistics by Elena Semino and Mick Short; (d) New-Dialect Formation by Peter Trudgill; and (e) Text, Context, Pretext by Henry G. Widdowson. One hundred tokens of each verb (CAN, COULD, MAY, MIGHT) assigned a possibility reading have been analysed; the tokens conveying other meanings than epistemic or root possibility have been excluded, being beyond the scope of the analysis. Due to the extent of the material and its character, the paper does not intend to suggest any general conclusions. It only attempts to fully exploit the material under investigation and thus draw attention to certain issues regarding the use of the examined modal verbs in the excerpted texts. 


\section{Epistemic vs. root possibility}

Before discussing the analysed data, the major difference between epistemic and root possibility will be revised. Epistemic possibility, conveying the speaker's lack of confidence in the truth of the proposition, involves subjectivity (Lyons 1977: 739), as in (1), where the speaker's subjective viewpoint and logical inference are apparent. On the other hand, the root possibility reading usually relates to statements of fact and is associated with external enabling/ disabling conditions (Bybee \& Fleischman 1995: 5), as in (2), which indicates that particular conditions (the fact that news reports are normally written in the third-person form) do not make the action possible.

(1) For example, the instructor's use of 'that' in the discussion of the final essay in Text Sample 3.4 may not be entirely clear. [BC, 65] $=\mathrm{I}$ think/it is possible that the instructor's use of 'that' is not entirely clear

(2) As news reports are normally only written in the third-person form, this further subdivision could not be made with respect to the newspaper data. [SS, 19]

= it was not possible to make this subdivision due to the fact that news reports are written...

Additionally, the two readings can be distinguished with regard to scope of negation, time reference or hypothetical marking (cf. Coates 1995, Tárnyiková 1978). Silva-Corvalán (1995: 74-75) explains that it is the main predication that is affected in case of epistemic modality and thus (1) reads it is possible that the use is not entirely clear. By contrast, Example (2) reads it was not possible (for us) to make this subdivision, which indicates that the modal predication is affected by negation and time reference in case of root modality (Coates 1983: 20).

The findings in Table 1 show that in the excerpted linguistic texts the analysed modal verbs are commonly employed to express root possibility (61\%). The modal verbs MAY and MIGHT, unlike CAN and COULD, regularly express both epistemic and root possibility, the latter being, as Coates (1995: 60) claims, restricted to formal language. Epistemic possibility is generally considered to be primary and the most common sense of MAY/MIGHT (Biber et al. 1999, Coates 1983, Leech 2004, Peters 2004), which has been confirmed, but the findings also indicate that in written academic texts root possibility MAY and MIGHT seem to be commonly employed (31\%). 
Exploring Modal Verbs Conveying Possibility in Academic Discourse

\begin{tabular}{lcccc}
\hline & Epistemic & Root & Indeterminate & Total \\
\hline CAN & - & 96 & 4 & 100 \\
COULD & - & 84 & 16 & 100 \\
MAY & 63 & 25 & 12 & 100 \\
MIGHT & 47 & 38 & 15 & 100 \\
Total & $110(27 \%)$ & $243(61 \%)$ & $47(12 \%)$ & 400 \\
\hline
\end{tabular}

Table 1: Possibility readings of the modal verbs in the excerpted texts

\subsection{Indeterminate cases}

Forty-seven occurrences (12\%) have been classified as indeterminate in that it was difficult to identify their appropriate reading even if contextual clues were provided.

All the indeterminate cases of CAN (4 occurrences) and three indeterminate cases of COULD in the excerpted material can be interpreted as relating the possibility and ability sense. In these cases, the fulfilment of the action seems to depend on a mixture of external factors and inherent properties of the subject (Leech \& Coates 1980), as in (3), which might read it is in our capacity to add or we will add due to external conditions.

(3) To this New Zealand evidence, we can also add some data from Australia... [PT, 18]

$=$ it is possible for us to add / we are able to add

As Leech and Coates (1980) state, ability implies possibility, which means that if someone has the ability to do something, then it is possible. Such cases can therefore be characterized in terms of 'gradience', specifically the gradient of inherency linking examples intermediate between ability and possibility (Coates 1983: 93).

On the other hand, all the indeterminate cases of MAY/MIGHT (27 instances) and the majority of indeterminate occurrences of COULD (13 instances) can be discussed in terms of 'merger' (Leech \& Coates 1980), which is restricted to formal written language and can be termed 'contextual neutralisation' (Coates 1995). The cases of merger (40 instances, i.e. 10\%) can be thus viewed as containing elements of both root and epistemic possibility: 
(4) This perception may be triggered by contextual factors such as the reader's knowledge about the opinions and intentions of the character, or his/her individual speech style. [SS, 27]

$=$ it is possible that this perception will be triggered by... (epistemic)

$=\mathrm{it}$ is possible for this perception to be triggered by .../ contextual factors enable to trigger this perception (root)

(5) One culture might perceive relatively small differences in purpose to be manifestations of different registers and/or genres, while another culture could perceive the same range of communicative events to all represent a single genre/register. [BC, 36]

$=$ it is possible that one culture will perceive..., while another culture will perhaps perceive... (epistemic)

= it would be possible for one culture to perceive..., while it would be possible for another culture to perceive... (root)

As the paraphrases suggest, it is not clear whether the authors convey their lack of confidence in the truth of the proposition or whether they present alternative possibilities. These examples indicate that the two interpretations, epistemic and root possibility, normally seen as unrelated categories, are not mutually exclusive (Coates 1983: 16) but tend to merge in academic discourse.

\section{Epistemic MAY and MIGHT}

As stated, MAY and MIGHT can express both epistemic and root possibility, the former being their primary and most common sense (55\% of MAY/MIGHT occurrences in the analysed texts) (cf. Coates 1983, Facchinetti 2003, Leech 2004). Coates (1983: 137) argues that the root-epistemic distinction of possibility MAY/MIGHT is associated with specific syntactic restrictions and contextual features and, as has been mentioned in Section 2, can be distinguished in terms of scope. Negated MAY and MIGHT seem to be associated exclusively with epistemic possibility (cf. Biber et al. 1999, Dušková 1972, Huddleston \& Pullum 2002, Leech 2004, or Tárnyiková 1978); all the analysed negated occurrences (13 instances) are interpreted as epistemic, for example (6), which illustrates that although the modal is morphologically marked for negation, it is the main predication that is affected, whereas the speaker's assessment of the proposition remains unaffected (cf. Coates 1983: 134, Facchinetti 2003: 312). Similarly, although the possible event in the past has to be indicated by MAY/MIGHT + perfect infinitive, the modal predication remains again unaffected, as in (7), where, in Leech's words, "the modal expresses a current state of the mind, while the main verb describes an event or state having variable time and aspect" (2004: 99). 
(6) This, he suggests, might not actually be an injunction, but a statement of general truth about gentlemen and their habitual behaviour, or even a loyal toast. [HGW, 12]

$=$ it is possible that this is not actually an injunction

(7) This is very important evidence because it is very likely that many features of the accent current in London in 1800, although they may subsequently have been lost from London English itself, were still current in rural East Anglia in 1870. [PT, 47]

$=$ it is possible that many features of the accent were lost...

It is also claimed that 100 per cent associations can be established for epistemic MAY when it co-occurs with an inanimate subject or with the pronoun it. However, this criterion focuses on distinguishing epistemic possibility from root permission and does not seem to relate to the differences between the two possibility readings. Both root and epistemic possibility MAY commonly co-occur with an inanimate subject (cf. examples in Section 4).

The majority of epistemic possibility readings (73\%) refer to the present, as in (6), and tend to co-occur with other epistemic devices signalling uncertainty, for example, likely, probably, seem. Moreover, epistemic MAY has been found to occur commonly in concessive clauses (one-third of epistemic MAY tokens), as in (7) or (8):

(8) In these cases, although the group of addressees may be very large, it is possible to identify who they are. [BC, 41]

$=$ although the group is perhaps large / although it is possible that the group is large

Such instances do not appear to convey the prototypical epistemic reading in that they indicate that a certain assumption is not ruled out. Quirk et al. (1985: 224) explain that the proposition in the concessive clause is presumed to be true and MAY is preferred to the alternative simple present as the proposition seems to be more tentative than its unmodalized counterpart. Huddleston and Pullum (2002: $179,182)$ claim that "it is difficult to detect any meaning difference between the unmodalized and modalized utterance" but they state that "the concessive adjunct reinforces the possibility meaning of MAY" (2002: 182). Papafragou (2000) justifies the epistemic interpretation of such examples, arguing that "MAY has its normal epistemic interpretation, its complement is an assumption which is derived by deliberate inferencing, and as such has come to belong to the speaker's 
belief-box with a degree of strength attached to it" (2000: 133).

The last brief comment concerns the semantic difference between epistemic MAY and MIGHT, which is generally associated with degrees of the speaker's certainty about the truth of the proposition (cf. Dušková 1994: 192, Huddleston 1993: 170, Palmer 1990: 58), as in (9):

(9) Or you may be interested in only research articles, a more specific register within academic prose. Or you might focus on medical research articles, or even only the methods sections of experimental medical research articles. [BC, 25]

Example (9) illustrates the use of MAY and MIGHT in similar contexts, showing the same range of time reference, but MIGHT seems to be the tentative form expressing a weaker degree of likelihood than MAY (cf. Leech 2004).

\section{Root possibility CAN and MAY}

This section focuses on discussing the major meaning recorded in the excerpted material, root possibility, primarily conveyed by CAN ( 96 tokens out of 100). As for root possibility MAY, Leech's study (2003) suggests: “...this sense was virtually absent from speech even in 1961 and has declined in writing since then, which can be assigned to the fact that it can be replaced by CAN" (2003: 234). However, the analysis reveals that the root possibility sense of MAY is employed in the excerpted academic discourse (25 tokens out of 100). Similarly, Leech and Coates's (1980: 86) findings prove that in formal written language the occurrence of root possibility MAY is significant even if this reading is often ignored or "regarded as a rarity of formal literary style".

Root possibility CAN and MAY typically occur in general statements, for example, sometimes sentences can be formally ambiguous; both terms may refer to a unit of language longer than the sentence. These examples indicate that the modals convey theoretical possibility and, unlike epistemic possibility, the attitude to the truth of the proposition is not involved here as the writers report a state of fact and draw conclusions on the basis of experiments or observations:

(10) They [texts] can correspond in extent with any linguistic unit: letter, sound, word, sentence, combination of sentences. [HGW, 8]

$=$ it is possible for texts to correspond with / some texts correspond with sound, word,... 
(11) For here they are units of actual written performance bounded by a capital letter and a full stop, which may (though, as we have seen need not) correspond with any number of units which can be analysed into sentences in the syntactic sense. [HGW, 14]

$=$ it is possible for the units to correspond with / it is sometimes the case that the units correspond with...

Examples (10) and (11) also illustrate that root CAN and MAY convey a kind of possibility which is termed 'existential possibility', i.e. what is sometimes the case or what applies to at least some members of the set (Palmer 1990: 152-154). Such instances can be paraphrased with expressions of quantification like some, any, all or sometimes because existential possibility involves generalization in that it applies to at least some members of the set.

One of the most important syntactic correlations for root possibility CAN and MAY seems to be the passive; passive structures have been recorded in 44 per cent of instances. As in Examples (12) and (13), passive structures with root possibility CAN and MAY convey what is circumstantially possible and mostly co-occur with third person inanimate subjects and unexpressed agents, usually implied in the context.

(12) At this point, it is simply important to realize that registers can be studied on many different levels of specificity. [BC, 10]

$=$ it is possible (for us) to study registers on many different levels of specificity

(13) Alternatively, theme and rheme may be associated with given and new. [HGW, 29]

$=$ it is possible (for us) to associate theme and rheme with given and new

Examples (10)-(13) also demonstrate that root possibility CAN and MAY tend to occur in similar contexts and thus sometimes appear to be interchangeable. For example, in (10) and (11) above, there does not seem to be much difference in meaning between CAN and MAY; they occur in analogous contexts, i.e. they are followed by the same lexical verb and convey theoretical possibility. Similarly, both verbs are employed in analogous passive structures like can be used/may be used, can be found/may be found. It might be concluded that in academic linguistic texts authors sometimes use root possibility CAN and MAY in affirmative structures interchangeably and thus the two verbs could be viewed as stylistic variants, MAY being the more formal equivalent to CAN (cf. Dušková 1994: 186, Leech 2004: 76). However, it should be noted that, generally, these two verbs 
can rarely be treated as synonymous because they are associated with different meanings and distinct usages, epistemic MAY vs. root CAN.

\section{Root possibility COULD and MIGHT}

Both COULD and MIGHT can convey epistemic and root possibility, however, with dissimilar frequency. The primary use of MIGHT is epistemic $(47 \%$ in the excerpted texts) but its root hypothetical possibility reading, viewed as restricted to formal language, is also significant $(38 \%)$. On the other hand, Could is essentially associated with root possibility ( $84 \%$ in the excerpted texts), whereas its less frequent epistemic possibility reading appears, as Coates (1983: 167) claims, mainly in spoken language; epistemic CouLD has not been identified in the analysed texts.

Huddleston and Pullum (2002: 196) characterize three major uses of the morphologically past tense forms of modals, namely past time, backshift and modal remoteness. They observe that the preterite forms predominantly appear in non-past contexts and Bybee (1995) adds that past modals can be considered to be unmarked for tense in that they express the meaning similar to their present counterparts, but weakened by hypotheticality. The findings prove that the majority of root possibility COULD and MIGHT tokens appear in non-past contexts (91 out of 122 , i.e. $75 \%$ ), where they can be interpreted as hypothetical root possibility:

(14) The number of possible contexts this expression could relate to is infinite, but we can narrow them down by co-textual extension. [HGW, 70]

$=$ it would be possible for this expression to relate to the infinite number of contexts

(15) Similarly in spoken language, a joke told during a sermon might be considered as a complete text, but the entire sermon can also be treated as a complete text. [BC, 5]

$=$ it would be possible (for us) to consider a joke to be a complete text

Examples (14) and (15) illustrate that the modals are associated with settings discussing potential states or situations where the writers draw tentative conclusions on the basis of experiments or observations. The interchangeability of COULD and MIGHT in such contexts seems to be possible, it is analogical to CAN and MAY (cf. Section 4). Like root possibility CAN and MAY, COULD and MIGHT can often be found in agentless passive structures (could be described, could be employed, might be analysed, might be considered), which are more tentative in comparison with those employing their present counterparts. Bybee 
and Fleischman (1995) summarize as follows: "When agent-oriented modals combine with past tense, the resulting unit often undergoes a meaning change, losing the past-tense component of its meaning and coming to signal a weakened version of its original modal meaning in the present tense" (1995: 12).

Additionally, hypothetical root MIGHT and COULD commonly occur with verbs such as argue, call, define, for example, we could argue, we might call, it could be defined, it might be objected, it might be argued. Leech (2004: 77) views such structures as "rather empty formulae soliciting and focusing the reader's attention", whose function is to draw the reader's attention and at the same time soften the force of what is being said in that the writer allows for the existence of alternative descriptions (Coates 1983: 160, Tárnyiková 1985: 40).

As for past time reference, with MIGHT it is signalled predominantly only in reported speech, while COULD is regularly used as the past form of CAN (Huddleston \& Pullum 2002: 109, 202) but is infrequent in comparison with hypothetical COULD. All the instances of past possibility COULD (20 tokens) occur in contexts which clearly indicate past setting, as in (16), where past context is indicated by the verb form needed:

(16) Since the messages needed to be short, only the "gist" could be conveyed. [BC, 204]

= it was possible to convey only the "gist"

Past time reference of root COULD is also conveyed by COULD in conjunction with perfect infinitive (9 instances), which refers to situations which could happen in the past. Four tokens appear in the context which implies the nonfulfilment of the possible action (cf. Dušková 1972: 22), as in (17) clearly stating it was not said, whereas the remaining five occurrences just refer to a possible situation in the past, as in (18):

(17) ...since there are different options to choose from within the grammatical systems, analysis can reveal what could have been said but was not. [HGW, 98]

$=$ it would have been possible to say something but it was not said

(18) This is so even though only between $4 \%$ and $8 \%$ of the colonists came from the southern langue d'oc area and could therefore have been uncontroversially described as being speakers of a language other than French. [PT, 7]

$=$ it was possible to describe the colonists as being speakers of a language other than French 
In summary, depending on the context, the structure COULD + perfect infinitive can be interpreted as denoting a possible situation in the past or a non-actualised event.

\section{Hedging}

Since modality is viewed as "the most important concept that cuts across the area of hedges" (Markkanen \& Schröder 1997: 5), this section discusses the potential use of the analysed modal verbs as hedging devices.

Hedges are generally taken to be modifiers of the speaker's commitment to the truth-value of a proposition, qualifying utterances to mitigate their strength. This viewpoint is held by Hyland (1995: 34), who defines hedges as various grammatical devices essentially representing tentativeness or an absence of certainty, employed to express claims with certain degree of caution, modesty and humility. Similarly, according to Biber et al. (1999) or Crompton (1997), the crucial role of hedges is to "explicitly qualify author's lack of knowledge to the truth of the proposition he utters" (1997: 273), which implies that hedging devices are closely related to epistemic possibility senses, where the uncertainty is identified with the speaker (cf. Section 3). Consequently, all the occurrences of epistemic MAY and MIGHT (110 instances) can be treated as the prototypical hedging forms toning down the speaker's commitment to the truth-value of the proposition.

Although it is epistemic modality that is primarily associated with hedging, the root meanings of modals may allow interpretation as hedges in certain contexts as well. In written academic discourse, hedges enable writers not only to present uncertain scientific claims based on their reasoning rather than certain knowledge (Hyland 1995: 34), they also help them avoid personal responsibility for statements, which may be achieved by employing various impersonal structures. This implies that although root possibility CAN and MAY are generally considered to be incompatible with the concept of hedging (cf. Hyland 1998 or Markkanen \& Schröder 1997), particularly when they convey existential possibility, their use in passive structures (can be regarded, can be explained, may be found, may be used) could be seen as writer-oriented hedging, which is, according to Hyland (1998), associated with depersonalization strategy, i.e. diminishing the role of the author. Furthermore, Preisler (1986: 92) argues that root modals can be given "interpersonal significance by the particular context in which they appear, usually as part of a tentativeness strategy" (cited in Markkanen \& Schröder 1997: 4). As illustrated in Section 5, root possibility COULD and MIGHT in non-past contexts usually convey tentative and speculative hypothetical possibility and could be thus viewed as hedges, for example, in (19) and (20) or in (14) and (15) above. 
(19) We therefore have no solid information which might lead us to any satisfactory indication of its dating. [PT, 81]

(20) Similarly, we are aware that our corpus could usefully comprise more text types and more samples within each text-type. [SS, 40]

To conclude, hedging does not seem to be restricted only to epistemic modal meanings. In the excerpted academic texts, root possibility CAN/MAY recorded in passive structures (53 occurrences) and COULD/MIGHT conveying hypothetical possibility in non-past contexts (91 occurrences) may function as hedges (cf. Hyland 1998). In sum, nearly 75 per cent of the analysed modal verbs could be viewed as hedging devices.

\section{Conclusion}

This small-scale investigation has revealed that the modal verbs CAN/COULD and MAY/MIGHT predominantly mark root possibility in the analysed linguistic texts. Root possibility MAY seems to be associated with formal written language because, in comparison with root possibility CAN, it conveys information more formally. Apart from presenting alternative possibilities, MAY and MIGHT in their primary epistemic sense also typically qualify statements. Facchinetti (2003) notes that "balancing between reporting objective data and signalling subjective evaluation seems to be fundamental in scientific discourse" (2003: 308).

Whereas MAY can convey both epistemic and root possibility, CAN does not seem to have any genuine epistemic uses. It possesses one underlying root sense, potentiality, and its possibility, ability or permission interpretations can be seen as contextually inferred in the appropriate settings. The analysis has also revealed that epistemic and root possibility senses of MAY/MIGHT (COULD), normally viewed as distinct and mutually exclusive, were difficult to separate in the analysed texts and could be thus interpreted in terms of merger. Then, it has been confirmed that COULD and MIGHT, primarily morphologically past tense forms, commonly occur in hypothetical uses in non-past contexts and can be described as tentative forms of CAN and MAY, unmarked for tense.

As for hedging, the examined modal verbs seem to be closely related to the concept of hedging, which is not restricted only to epistemic modal meanings. There are certain uses of root possibility readings, particularly those of hypothetical COULD/MIGHT, which satisfy the criteria defining hedges. On the other hand, CAN and MAY in active structures, primarily associated with theoretical possibility, do not normally hedge utterances in that they do not weaken the strength of the assertion. 
Finally, it is necessary to emphasize that the results of the analysis and the validity of the suggested interpretations are limited by the extent of the sample, which is not sufficient to draw any general conclusions. Bearing in mind that the investigation of more diverse material would be required, this paper only draws some tentative conclusions concerning the use of the modal verbs CAN/COULD and MAY/MIGHT in the excerpted academic linguistic texts.

\section{References}

Biber, D., Johansson, S., Leech, G., Conrad, S. and Finegan, E. (1999) Longman Grammar of Spoken and Written English. London: Longman.

Bybee, J. L. and Fleischman, S. (eds) (1995) Modality in Grammar and Discourse. Amsterdam and Philadelphia: John Benjamins.

Bybee, J. L. (1995) 'The semantic development of past tense modals in English.' In: Bybee, J. L. and Fleischman, S. (eds) Modality in Grammar and Discourse. Amsterdam and Philadelphia: John Benjamins. 503-517.

Coates, J. (1983) The Semantics of the Modal Auxiliaries. London: Croom Helm.

Coates, J. (1995) 'The expression of root and epistemic possibility in English.' In: Bybee, J. L. and Fleischman, S. (eds) Modality in Grammar and Discourse. Amsterdam and Philadelphia: John Benjamins. 55-66.

Crompton, P. (1997) 'Hedging in academic writing: Some theoretical problems.' English for Specific Purposes 16/4, 271-287.

Dušková, L. (1972) 'A contrastive study of can, may, must and their Czech equivalents.' Studies in Modern Philology 1, 5-77.

Dušková, L. (1994) Mluvnice současné angličtiny na pozadí češtiny. Praha: Academia.

Facchinetti, R. (2003) 'Pragmatic and sociological constraints on the functions of may in contemporary British English.' In: Facchinetti, R., Krug, M. and Palmer, F. R. (eds) Modality in Contemporary English. Berlin: Mouton de Gruyter. 301-327.

Huddleston, R. (1993) Introduction to the Grammar of English. Cambridge: Cambridge University Press.

Huddleston, R. and Pullum, G. (2002) The Cambridge Grammar of the English Language. Cambridge: Cambridge University Press.

Hyland, K. (1995) 'The author in the text: Hedging scientific writing.' Hong Kong Papers in Linguistics and Language Teaching 18, 33-42.

Hyland, K. (1998) Hedging in Scientific Research Articles. Amsterdam: John Benjamins. Leech, G. (2003) 'Modality on the move: The English modal auxiliaries 1961-1992.' In: Facchinetti, R., Krug, K. and Palmer, F. R. (eds) Modality in Contemporary English. Berlin: Mouton de Gruyter. 223-240.

Leech, G. (2004) Meaning and the English Verb. London: Longman.

Leech, G. and Coates, J. (1980) 'Semantic indeterminacy and the modals.' In: Quirk, R., Greenbaum, S., Leech, G. and Svartvik, J. (eds) Studies in English Linguistics. London: Longman. 79-99.

Lyons, J. (1977) Semantics. Cambridge: Cambridge University Press.

Markkanen, R. and Schröder, H. (1997) 'Hedging: A challenge for pragmatics and discourse analysis.' In: Markkanen, R. and Schröder, H. (eds) Hedging and Discourse: Approaches to the Analysis of a Pragmatic Phenomenon in Academic Texts. Berlin and New York: Walter de Gruyter. 3-20. 
Palmer, F. R. (1990) Modality and the English Modals. London: Longman.

Papafragou, A. (2000) Modality: Issues in the Semantics-Pragmatics Interface. Oxford: Elsevier.

Peters, P. (2004) The Cambridge Guide to English Usage. Cambridge: Cambridge University Press.

Quirk, R., Greenbaum, S., Leech, G, and Svartvik, J. (1985) A Comprehensive Grammar of the English Language. London: Longman.

Silva-Corvalán, C. (1995) 'Interpretation of poder and deber.' In: Bybee, J. L. and Fleischman, S. (eds) Modality in Grammar and Discourse. Amsterdam and Philadelphia: John Benjamins. 67-105.

Tárnyiková, J. (1978) 'Semantic components of some modal verbs.' Germanica Olomucensia 4, 7-22.

Tárnyiková, J. (1985) Modalita v angličtině (část 1). Olomouc: Univerzita Palackého.

\section{Sources}

Biber, D. and Conrad, S. (2009) Register, Genre and Style. Cambridge: Cambridge University Press. [BC]

Blakemore, D. (2002) Relevance and Linguistic Meaning: The Semantics and Pragmatics of Discourse Markers. Cambridge: Cambridge University Press. [DB]

Semino, E. and Short, M. (2004) Corpus Stylistics: Speech, Writing and Thought Presentation in a Corpus of English Writing. London: Routledge. [SS]

Trudgill, P. (2004) New-Dialect Formation: The Inevitability of Colonial Englishes. Edinburgh: Edinburgh University Press. [PT]

Widdowson, H. G. (2004) Text, Context, Pretext: Critical Issues in Discourse Analysis. Oxford: Blackwell. [HGW]

Petra Huschová is Assistant Professor of English Linguistics at the University of Pardubice, Czech Republic. Her dissertation dealt with the English modal verbs expressing possibility in written English (Epistemic and Root Possibility Meanings of CAN and MAY in Written English). She specialises in stylistics and pragmatics, focusing mainly on academic discourse, hedging and modality.

Address: PhDr. Petra Huschová, Ph.D., Department of English and American Studies, Faculty of Arts and Philosophy, University of Pardubice, Studentská 84, 53210 Pardubice, Czech Republic. [e-mail: petra.huschova@upce.cz] 